Alex Sandro Rolland Souza'

ADRIANA SCAVUZZI ${ }^{2}$

David Coelho Rodrigues ${ }^{3}$

Roberta Dantas de Oliverira ${ }^{4}$

Francisco Edson LuCena Feitosa ${ }^{5}$

Melania Maria Ramos Amorim ${ }^{6}$

\section{Solução oral escalonada de misoprostol para indução do parto: estudo piloto}

\author{
Titrated oral solution of misoprostol for labour induction: a pilot study
}

Artigo original

Palavras-chave

Trabalho de parto Trabalho de parto induzido Misoprostol/administração \& dosagem Administração oral

Estudo multicêntrico

Ensaio clínico

Keywords

Labor, obstetric Labor, induced Misoprostol/administration \& dosage Administration, oral Multicenter study Clinical trial

Correspondência:

Alex Sandro Rolland de Souzo Avenida Rui Barbosa, 579, apto. 406 - Graças CEP 52011-040 - Recife (PE), Brasil E-mail: alexrolland@uol.com.br

Recebido

$15 / 3 / 10$

Aceito com modificacõos

$22 / 4 / 10$

\section{Resumo}

OBJETIVO: avaliar a efetividade e a segurança da administração de uma nova formulação de misoprostol em solução por via oral, com doses escalonadas, para indução do parto de feto vivo a termo. MÉTODOS: realizou-se um estudo multicêntrico, do tipo ensaio clínico, aberto, não-randomizado, no período de Julho a Dezembro de 2008. Foram incluídas 30 pacientes com indicação de indução do trabalho de parto, a termo, com feto vivo, índice de Bishop $\leq 6$, apresentação cefálica, peso fetal estimado pela ultrassonografia $<4.000 \mathrm{~g}$ e índice de líquido amniótico $>5$. Foram excluídas mulheres com cicatriz uterina, cardiotocografia alterada, gestação múltipla, restrição de crescimento fetal, hemorragia genital e presença de tumores, ulcerações ou malformações genitais. A dose inicial da solução oral foi de $20 \mu \mathrm{g} / \mathrm{h}$ de misoprostol, nas primeiras 6 horas, aumentando em $20 \mu \mathrm{g} / \mathrm{h}$ de misoprostol a cada 6 horas, se o trabalho de parto não fosse deflagrado, até uma dose máxima de $80 \mu \mathrm{g} / \mathrm{h}$, nas primeiras 24 horas, mantendo a dose máxima $(80 \mathrm{\mu g} / \mathrm{h})$ por mais 24 horas, se necessário. RESULTADOS: o trabalho de parto foi induzido satisfatoriamente

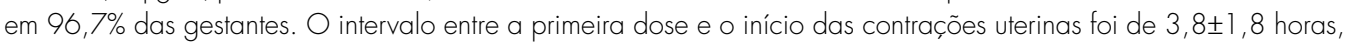
enquanto o intervalo entre a dose inicial e o parto variou entre 6 e 24 horas. A frequência de parto vaginal foi de $80 \%(n=24)$. A maioria das gestantes iniciou o trabalho de parto com a dose de $20 \mu \mathrm{g} / \mathrm{h}(60 \% ; n=18)$. A taquissistolia ocorreu em 13,3\% das gestações e líquido meconial foi detectado em 20\% dos casos. Houve dois casos de escore de Apgar < 7 no primeiro minuto e nenhum no quinto minuto. CONCLUSÕES: a solução oral de misoprostol administrada de forma escalonada foi efetiva e segura para indução do trabalho de parto. No entanto, são necessários estudos controlados para comparação com a via vaginal.

ClinicalTrial.gov Identifier: NCT00992524

\section{Abstract}

PURPOSE: to test effectiveness and safety of the oral administration of a new misoprostol formulation in titrated doses for the induction of delivery of a live fetus at term. METHODS: an open pilot multicenter, non-randomized clinical trial was conducted from July to December 2008. A total of 30 patients with indications for induction of labor were included. The patients had a live fetus, Bishop score $\leq 6$, vertex presentation, fetal weight $<4,000 \mathrm{~g}$ estimated by ultrasonography and amniotic fluid index $>5$. Exclusion criteria were previous uterine scar, non-reassuring fetal heart rate tracing, multiple pregnancy, fetal growth restriction, genital hemorrhage and presence of genital tumors, ulcerations or malformations. An initial dose of $20 \mathrm{\mu g} /$ hour of the oral misoprostol solution was used in the first 6 hours, and was increased progressively to $20 \mathrm{\mu g} /$ hour every 6 hours if labor did not start, up to a maximum dose of $80 \mathrm{\mu g} / \mathrm{h}$ in the first 24 hours, maintained for additional 24 hours if necessary. RESULTS: labor was satisfactorily induced in $96.7 \%$ of patients. The interval between

Centro de Atenção à Mulher do Instituto de Medicina Integral Prof. Fernando Figueira - IMIP - Recife (PE), Brasil. Instituto de Saúde Elpídio de Almeida - ISEA - Campina Grande (PB), Brasil.

Maternidade Escola Assis Chateaubriand da Universidade Federal do Ceará - UFC - Fortaleza (CE), Brasil.

' Pós-graduando (Doutorado) em Saúde Materno Infantil do Instituto de Medicina Integral Prof. Fernando Figueira - IMIP - Recife (PE), Brasil; Supervisor da Residência Médica e do Setor em Medicina Fetal do Instituto de Medicina Integral Prof. Fernando Figueira IMIP - Recife (PE), Brasil.

2 Pós-graduando (Doutorado) em Saúde Materno Infantil do Instituto de Medicina Integral Prof. Fernando Figueira - IMIP - Recife (PE), Brasil; Preceptora da Residência Médica em Ginecologia e Obstetrícia do Instituto de Medicina Integral Prof. Fernando Figueira IMIP - Recife (PE), Brasil.

${ }^{3}$ Médico (a) Residente em Ginecologia e Obstetrícia da Maternidade Escola Assis Chateaubriand da Universidade Federal do Ceará - UFC - Fortaleza (CE), Brasil.

${ }^{4}$ Médico (a) Residente em Ginecologia e Obstetrícia da Maternidade Escola Assis Chateaubriand da Universidade Federal do Ceará - UFC - Fortaleza (CE), Brasil.

${ }^{5}$ Coordenador da Residência Médica em Tocoginecologia da Maternidade Escola Assis Chateaubriand da Universidade Federal do Ceará - UFC - Fortaleza (CE), Brasil.

- Professora da Pós-graduação em Saúde Materno Infantil do Instituto de Medicina Integral Prof. Fernando Figueira - IMIP - Recife (PE), Brasil; Médica Obstetra do Instituto de Saúde Elpídio de Almeida - ISEA - Campina Grande (PB), Brasil.

Conflito de interesse: a pedido dos pesquisadores, a solução oral foi preparada exclusivamente para a pesquisa no Laboratório

Hebron, a qual foi adquirida com recursos próprios dos mesmos. 
the first dose and the beginning of uterine contractions was 3.8 1.8 hours. The interval between the initial dose and delivery varied from 6 to 24 hours. The frequency of vaginal delivery was $80 \%$ (24 cases). Most of the patients $(60 \% ; n=18)$ initiated labor with a dose of $20 \mu \mathrm{g} /$ hour. Tachysystole occurred in $13.3 \%$ of women and meconium-stained fluid was detected in $20 \%$ of cases. There were two cases of Apgar scores $<7$ in the first minute and no Apgar score $<7$ in the fifth minute. CONCLUSIONS: the oral solution of misoprostol was effective and safe for the induction of labor. However, further randomized controlled trials are needed to compare this new formulation with misoprostol administered by the vaginal route.

ClinicalTrial.gov Identifier: NCT00992524

\section{Introdução}

O misoprostol, um análogo sintético da prostaglandina E1 (PGE1), vem sendo largamente utilizado em Obstetrícia, particularmente para indução do trabalho de parto, desde $1991^{1,2}$.

Vários estudos avaliaram a efetividade e a segurança do misoprostol por diferentes vias e formas de administração (oral, bucal, sublingual e vaginal) $)^{3-5}$. Atualmente, diretrizes de diversas sociedades em todo o mundo recomendam principalmente o uso do misoprostol por via vaginal $^{6,7}$. No entanto, outras formas de administração vêm sendo estudadas, como oral e sublingual, por serem menos incômodas e consideradas mais aceitáveis para as pacientes $^{4,8}$.

Estudos avaliando a farmacocinética dessa droga de acordo com as diferentes vias de administração observaram que o pico de concentração plasmática da droga é maior entre as grávidas que recebem a droga por via sublingual que a oral, vaginal com adição de água e vaginal. A biodisponibilidade foi também maior com o uso da via sublingual. Entretanto, o nível plasmático foi mantido por um maior período de tempo quando a via vaginal foi utilizada ${ }^{9}$.

Assim, pelo fato de a via oral ser bem aceita pelas pacientes, por diminuir o número de exames vaginais e apresentar um pico de concentração que se mantém por um período de tempo mais curto que a via vaginal, surgiram estudos utilizando uma baixa dose de misoprostol por curto intervalo de tempo, aumentando a dose, quando necessário $^{10}$.

A solução oral de misoprostol em doses escalonadas foi avaliada em um ensaio clínico randomizado, aberto, para indução do parto em gestações a termo com feto vivo. A solução oral de misoprostol foi preparada a partir do comprimido de $200 \mu \mathrm{g}$ diluído em $200 \mathrm{~mL}$ de água, correspondendo a $1 \mu \mathrm{g} / \mathrm{mL}$. O tratamento foi iniciado com dose de $20 \mathrm{~mL}$ a cada hora, durante 4 horas, podendo ser aumentada, se necessário, para $40 \mathrm{~mL}$, seguidos de $60 \mathrm{~mL}$ a cada hora, por mais 4 horas, com dose máxima cumulativa de $1.600 \mu \mathrm{g}$. O Grupo Controle recebeu o comprimido vaginal de $25 \mu \mathrm{g}$ a cada 4 horas. Os resultados foram bastante animadores, com uma frequência de parto vaginal em 12 horas de 74,3\% no Grupo da Solução Oral e de $25,5 \%$ no Grupo Vaginal, além de menor incidência da síndrome de hiperestimulação uterina no Grupo da Solução Oral ${ }^{10}$.

A literatura demonstra que o misoprostol é, na atualidade, o método mais efetivo para amadurecimento do colo e indução do parto, porém ainda é necessário definir qual a melhor e a menor dosagem capaz de desencadear o trabalho de parto, com baixa incidência de complicações, bem como qual a melhor via de administração $0^{3-8}$.

$\mathrm{Na}$ tentativa de contribuir para esclarecer essas dúvidas e diminuir os índices de cesárea em nosso meio, foi proposto este estudo piloto com o objetivo de determinar a efetividade da solução oral escalonada de misoprostol para indução do parto a termo com feto vivo, comparando-se os resultados ao único estudo encontrado na literatura com essa forma de administração.

\section{Métodos}

Realizou-se um estudo piloto multicêntrico, do tipo ensaio clínico aberto, envolvendo 30 gestantes de alto risco atendidas na Maternidade Escola Assis Chateaubriand da Universidade Federal do Ceará (UFC), Fortaleza (CE), no Instituto de Medicina Integral Professor Fernando Figueira (IMIP), Recife (PE), e no Instituto de Saúde Elpídio de Almeida (ISEA), em Campina Grande (PB), no período de Julho a Dezembro de 2008. Todos os serviços estão localizados no Nordeste do Brasil.

Este estudo foi aprovado pelos Comitês de Ética em Pesquisa das instituições. Todas as pacientes foram devidamente informadas sobre os objetivos da pesquisa e somente foram incluídas caso concordassem livremente em participar, assinando o termo de consentimento livre e esclarecido.

A solução oral de misoprostol foi preparada exclusivamente para esta pesquisa pelo laboratório Hebron (Caruaru, PE) na concentração de $1 \mu \mathrm{g} / \mathrm{mL}$, a pedido dos investigadores do presente estudo. Essa solução foi devidamente homogeneizada a partir do princípio ativo de misoprostol, sem que fosse necessária a sua preparação artesanal a partir do comprimido, o que abre novas perspectivas para o estudo dessa via de administração.

O cálculo do tamanho da amostra foi realizado no programa StatCalc do Epi-Info versão 3.5, prevendo-se um percentual de partos vaginais nas primeiras 24 horas em torno de $95 \%^{10}$, com uma precisão de $10 \%$ e um nível de confiança de $95 \%$. De acordo com esse cálculo, 
seriam necessárias 23 pacientes, número que foi aumentado para 30, prevendo-se eventuais perdas ou diferenças nessa frequência.

Foram incluídas pacientes com indicação para indução do trabalho de parto que se apresentassem com gestação única, idade gestacional superior a $37^{\mathrm{a}}$ semana de gravidez, feto vivo, em apresentação cefálica, peso fetal estimado por ultrassonografia menor que $4.000 \mathrm{~g}$, índice de líquido amniótico (ILA) maior que $5 \mathrm{~cm}$, cardiotocografia basal anteparto normal e índice de Bishop $\leq 6$.

Foram excluídas as gestantes com cicatriz uterina, apresentação anômala, provas de vitalidade fetal alteradas (cardiotocografia ou perfil biofísico fetal), restrição de crescimento fetal, gestação múltipla, malformação congênita, hemorragia vaginal e qualquer contraindicação para o parto vaginal, como tumores, malformação ou ulcerações genitais.

A idade gestacional foi estimada pela data da última menstruação, quando conhecida e confiável, e confirmada pela primeira ultrassonografia. A paciente foi submetida à cardiotocografia basal anteparto imediatamente antes do uso do misoprostol.

A administração do misoprostol foi realizada na Enfermaria de Gestação de alto risco, pelo médico assistente, o qual oferecia a solução oral a cada hora na dose inicial de $20 \mu \mathrm{g} / \mathrm{h}(20 \mathrm{~mL} / \mathrm{h})$ de misoprostol, nas primeiras seis horas. Esta dose era aumentada em $20 \mu \mathrm{g} / \mathrm{h}$ de misoprostol a cada 6 horas, se o trabalho de parto não fosse deflagrado, até uma dose máxima de $80 \mu \mathrm{g} / \mathrm{h}(80 \mathrm{~mL} / \mathrm{h})$ de misoprostol nas primeiras 24 horas, mantendo-se a dose máxima $(80 \mu \mathrm{g} / \mathrm{h})$ por mais 24 horas, se necessário.

As pacientes foram acompanhadas, monitorizandose a presença de contrações uterinas a cada hora, até o parto. O intervalo entre as doses foi aumentado a cada seis horas, até o máximo de $80 \mu \mathrm{g} / \mathrm{h}$, por um período de tempo máximo de 48 horas ou até o início do trabalho de parto. Considerou-se como padrão contrátil satisfatório a presença de pelo menos três contrações de 40 ou mais segundos de duração, em 10 minutos de observação. Após seis horas da administração da última dose do medicamento e na ausência de trabalho de parto, o tratamento era suspenso, e se considerou como falha de indução, sendo a cesariana indicada.

Após o início do trabalho de parto, as gestantes foram encaminhadas ao pré-parto e acompanhadas pelos médicos de plantão do centro obstétrico nas instituições, monitorizando-se a dinâmica uterina e a frequência cardíaca fetal (FCF). A ausculta fetal foi realizada de forma intermitente em todos os casos, a cada 15 minutos, antes, durante e depois das contrações, quando presentes. A dinâmica foi verificada a cada 30 minutos.

A idade materna, a idade gestacional, a paridade, o índice de Bishop inicial e as indicações para indução do parto foram as características avaliadas das gestantes incluídas no estudo.

As principais variáveis colhidas foram: intervalo transcorrido entre o início da indução (administração da primeira dose) e o início das contrações uterinas; intervalo entre o início das contrações e o parto; intervalo entre administração da primeira dose e o parto; frequência de mulheres que necessitaram da dose mínima de $20 \mu \mathrm{g} / \mathrm{h}$ para início do padrão contrátil satisfatório (início do trabalho de parto); indução do parto satisfatória, quando a paciente iniciou o padrão de contrações uterinas adequadas; taquissistolia; hipertonia; síndrome de hiperestimulação; e alterações da FCF durante o trabalho de parto.

Os desfechos avaliados após o parto foram: a presença de mecônio no líquido amniótico; escores de Apgar $<7$ no primeiro e no quinto minuto; óbito perinatal; tipo de parto (vaginal, cesariana ou fórceps); e indicação da cesariana. Foram ainda observados efeitos colaterais gastrintestinais, como náuseas, vômitos e diarreia.

Os distúrbios da contratilidade uterina foram definidos de acordo com o Royal College of Obstetricians and Gynaecologists (RCOG), considerando-se taquissistolia a presença de pelo menos cinco contrações uterinas por dois períodos consecutivos de dez minutos. A hipertonia foi caracterizada quando houve uma contração única de pelo menos dois minutos de duração. A síndrome de hiperestimulação foi definida na vigência de taquissistolia ou hipertonia uterina associada a padrões anômalos de FCF. Foram consideradas como alterações da frequência cardíaca bradicardia (quando frequência cardíaca fetal basal inferior a $110 \mathrm{bpm}$ ) e taquicardia (quando superior a $160 \mathrm{bpm}$ ) e/ou desacelerações tardias, tipo $\mathrm{II}^{7}$.

O tratamento da taquissistolia foi realizado com nifedipina na dosagem de $20 \mathrm{mg}$ por via sublingual, repetindo-se a cada 30 minutos, se necessário. Além disso, preconizava-se a adoção de decúbito lateral esquerdo e oxigenioterapia materna sob cateter a $5 \mathrm{~L} /$ minuto. $\mathrm{O}$ atendimento aos recém-nascidos seguiu as normas recomendadas em cada instituição.

Diante disso, o estudo foi realizado em 30 gestantes com indicação de indução do parto, a qual foi feita com a solução oral de misoprostol. A idade materna variou entre 18 e 36 anos, média de 24,5 55,6 anos. A mediana da paridade foi 0 , variando de 0 a 4 . A média da idade gestacional foi de 39,1 $\pm 1,8$ semanas. No momento da inclusão no estudo, o índice de Bishop variou de 0 a 6 , com mediana de 4 (Tabela 1). As principais indicações da indução do parto foram gravidez a partir da $41^{\text {a }}$ semana (40\%), síndromes hipertensivas da gestação $(36,7 \%)$ e rotura prematura das membranas a termo $(13,3 \%)$ (Tabela 2).

Os dados coletados foram devidamente registrados em formulário padrão, pré-codificados para entrada de dados 
Tabela 1 - Características das gestantes submetidas à indução do parto com solução oral de misoprostol

\begin{tabular}{lc}
\hline Característica & Valores \\
\hline Idade materna (variação) & $18-36$ anos \\
Idade maferna (média \pm DP) & $24,5 \pm 5,6$ anos \\
Paridade (variação) & $0-4$ \\
Paridade (mediana) & 0 \\
Idade gestacional (variação) & $37-42$ semanas \\
Idade gestacional (média \pm DP) & $39,1 \pm 1,8$ semanas \\
Índice de Bishop (variação) & $0-6$ \\
Índice de Bishop (mediana) & 4 \\
\hline DP: desvio padrão. &
\end{tabular}

Tabela 2 - Principais indicações da indução do parto com solução oral de misoprostol

\begin{tabular}{lcc}
\hline Indicações & n & $\%$ \\
\hline Gestação $\geq 41$ semanas & 12 & 40,0 \\
Síndromes hiperfensivas na gestação & 11 & 36,7 \\
Ruptura prematura das membranas a & 4 & 13,3 \\
termo & & \\
Diabetes gestacional & 2 & 6,7 \\
Outra & 1 & 3,3 \\
Total & 30 & 100,0 \\
\hline
\end{tabular}

em computador. Em seguida, foram digitados em banco de dados criado no programa estatístico, Epi-Info versão 3.5 para Windows. Para todas as variáveis, obtiveram-se tabelas de distribuição de frequência; medidas de tendência central e de dispersão foram calculadas, quando pertinentes.

\section{Resultados}

A indução do parto foi considerada satisfatória em 29 pacientes $(96,7 \%)$. Dessas, 24 mulheres $(80 \%)$ evoluíram para parto vaginal e 6 (20\%) para cesariana (Tabela 3). As principais indicações de cesárea foram falha de progressão (três casos) e desproporção céfalo-pélvica (dois casos). Apenas uma cesárea foi realizada por falha de indução.

$\mathrm{O}$ intervalo médio entre a primeira dose do misoprostol e o início do trabalho de parto foi de 3,8 $\pm 1,8$ horas, variando de 2 a 12 horas. O intervalo médio entre a primeira dose do misoprostol e o parto foi de $11,6 \pm 3,4$ horas, variando de 6 a 24 horas. Destaca-se que 18 pacientes $(60 \%)$ iniciaram o trabalho de parto com a dose mínima de $20 \mu \mathrm{g} / \mathrm{h}$. A taquisistolia ocorreu em quatro pacientes, a eliminação de mecônio em seis pacientes e escores de Apgar $<7$ no primeiro minuto foram observados em dois recém-nascidos. Não houve casos de hipertonia, síndrome de hiperestimulação uterina, alterações da FCF durante o trabalho de parto, escores de Apgar < 7e no quinto minuto e óbito perinatal (Tabela 3).
Tabela 3 - Desfechos maternos e neonatais das pacientes submetidas à inducão do parto com solucão oral de misoprostol.

\begin{tabular}{|c|c|}
\hline Desfechos & Valores \\
\hline $\begin{array}{l}\text { Intervalo da } 1^{a} \text { dose e início do traballho de parto em horas } \\
\text { (variação) }\end{array}$ & $2-12$ \\
\hline $\begin{array}{l}\text { Intervalo da } 1^{a} \text { dose e início do trabalho de parto em horas } \\
\text { (média } \pm \text { DP) }\end{array}$ & $3,8 \pm 1,8$ \\
\hline Intervalo da $1^{a}$ dose e parto em horas (variação) & $6-24$ \\
\hline Intervalo da $1^{a}$ dose e o parto em horas (média $\pm D P$ ) & $11,6 \pm 3,4$ \\
\hline Indução do parto satisfatória (n; \%) & $29 ; 96,7 \%$ \\
\hline Parto vaginal dentro de 24 horas $(n ; \%)$ & $24 ; 80 \%$ \\
\hline Cesariana* $(n ; \%)$ & $6 ; 20 \%$ \\
\hline $\begin{array}{l}\text { Número de casos em que a dose mínima }(20 \mu g) \text { foi necessária para } \\
\text { indução }(n ; \%)\end{array}$ & $18 ; 60 \%$ \\
\hline Taquisistolia (n; \%) & $4 ; 13,3 \%$ \\
\hline $\begin{array}{l}\text { Efeitos colaterais gastrointestinais (náusea, vômito e diarreia) } \\
(n ; \%)\end{array}$ & $2 ; 6,7 \%$ \\
\hline Mecônio (n; \%) & $6 ; 20 \%$ \\
\hline Escore de Apgar <7 no primeiro minuto (n; \%) & $2 ; 6,7 \%$ \\
\hline
\end{tabular}

\section{Discussão}

A indução do parto com misoprostol pela via oral merece destaque, por representar uma forma de administração de fácil manuseio e ser bastante aceitável pelas pacientes. Entretanto, a melhor dose ainda permanece incerta ${ }^{4}$. A forma escalonada de administração é baseada nos estudos farmacológicos da droga ${ }^{1,9}$, com a vantagem de manter constante a concentração do misoprostol no plasma materno ${ }^{10}$.

Revisando os bancos de dados Medline/Pubmed, Lilacs/ SciELO e da biblioteca Cochrane, com base nos descritores "misoprostol" e "trabalho de parto induzido", encontrouse um único estudo utilizando essa forma escalonada da solução, com resultados bastante animadores ${ }^{10}$. Assim, o nosso estudo, apesar de essencialmente descritivo, foi comparado, não apenas a essa forma escalonada de administração do misoprostol, mas também a outras vias.

O presente estudo foi um ensaio clínico randomizado aberto, com o objetivo de avaliar a efetividade da solução oral escalonada, constituindo um estudo piloto para um futuro ensaio clínico randomizado e triplo cego. Destacam-se as limitações desse estudo, pela ausência de um grupo controle, além do pequeno tamanho da amostra. A falta de um grupo controle com dose padronizada de misoprostol oral ou vaginal impede comprovar se existem diferenças significativas entre essa e outras formulações de misoprostol.

No entanto, julga-se pertinente a sua publicação, por ter sido este o primeiro estudo avaliando a efetividade de uma solução oral de misoprostol homogeneizada por 
um laboratório farmacêutico e preparada especificamente para a pesquisa. Cumpre destacar que, em se tratando de um estudo piloto com pequeno número de pacientes incluídas, algumas variáveis descritas em outros estudos e definidas como desfechos primários não puderam ser analisadas $^{2-5,8,10-14}$.

Um dos desfechos principais deste estudo foi o intervalo entre a primeira dose e o início do trabalho de parto, que foi consideravelmente maior que o descrito na literatura ${ }^{10}$. Enquanto neste estudo encontrou-se um intervalo médio de 3,8 horas para desencadear o trabalho de parto, esse intervalo, no estudo de Cheng et al. ${ }^{10}$, foi de 1,6 horas. Destaca-se que ambos os intervalos foram inferiores a outras vias e formas de administração do misoprostol, 5 horas na sublingual ${ }^{11}$ e 14 horas na vaginal ${ }^{12}$.

Além disso, o intervalo entre a primeira dose e o parto também foi diferente, encontrando-se uma média de aproximadamente 11,6 horas neste estudo, contra 8,2 horas no estudo de Cheng et al. ${ }^{10}$. Não se encontrou uma justificativa plausível para as grandes diferenças entre os dois estudos; apenas pode-se sugerir que o pequeno número da amostra pode ter influenciado nestes resultados. Estudo piloto realizado com a via sublingual observou o intervalo entre a primeira dose e o parto semelhante a esta pesquisa, aproximadamente 12 horas ${ }^{11}$. Porém, outro estudo observou intervalo de 22 horas com a via vaginal ${ }^{12}$. Sugere-se que essa diferença entre os resultados seja em razão da via de administração: a farmacologia da via sublingual seria mais parecida a da via oral do que à vaginal ${ }^{9}$.

Ressalta-se que os resultados descritos por Cheng et al. ${ }^{10}$ são bem diferentes do que descreve a literatura sobre misoprostol oral em baixas doses ${ }^{4}$, inclusive com uma taxa de 75\% de parto vaginal em 12 horas. Autores observaram que utilizando a via sublingual ou vaginal, o parto em 12 horas ocorreu em aproximadamente $26 \%$ das mulheres submetidas ao trabalho de parto induzido com misoprostol ${ }^{13}$.

Considerou-se indução satisfatória quando as pacientes entraram em trabalho de parto, independentemente da via do parto. Observou-se que a indução do parto foi adequada em 96,6\% das pacientes; apenas em uma paciente ocorreu falha de indução, sendo indicada a cesariana. $\mathrm{O}$ parto vaginal ocorreu em $80 \%$ das mulheres, variando o tempo entre administração do misoprostol e parto entre 6 e 24 horas. Entretanto, mais uma vez, no estudo de Cheng et al. ${ }^{10}$, observou-se uma taxa superior de parto vaginal (95\% de parto vaginal dentro de 24 horas e de $75 \%$ dentro de 12 horas $)^{10}$. Todavia, essa frequência descrita contrasta bastante com as descritas por outros autores utilizando misoprostol oral ${ }^{4,13}$. Na revisão sistemática disponibilizada na biblioteca Cochrane, que incluiu 10 ensaios clínicos comparando o misoprostol oral ao vaginal, encontrou-se uma frequência de parto vaginal não ocorrido dentro de
24 horas de $46 \%$ com o misoprostol oral, sem diferença significativa ${ }^{4}$. Percentual semelhante foi encontrado em outra revisão sistemática, que comparou a via sublingual à vaginal, também sem diferença estatística ${ }^{8}$.

No presente estudo, a cesariana foi necessária em seis pacientes (20\%), sendo as principais indicações falha de progressão (três casos) e desproporção céfalo-pélvica (dois casos). Apenas uma cesárea foi realizada por falha de indução. A taxa de cesariana descrita por Cheng et al. ${ }^{10}$ foi bem mais baixa, com apenas $4 \%$ de cesáreas no grupo que recebeu solução oral escalonada e $17 \%$ no grupo que recebeu misoprostol vaginal. Nesse estudo, foram relatadas apenas duas indicações de cesariana, falha de indução e frequência cardíaca fetal não-tranquilizadora, não sendo especificadas as outras. Entretanto, essa taxa de cesariana observada neste estudo de $20 \%$ encontra-se dentro da faixa descrita pela literatura, a qual corresponde a $20,7 \%$ com a via oral ${ }^{4}$ e $29 \%$ com a via sublingual ${ }^{8}$.

Em relação à falha de indução, atualmente não existe um consenso sobre os critérios para defini-la. Também não há consenso sobre em quanto tempo deve-se tentar a indução do parto. No estudo de Cheng et al. ${ }^{10}$, utilizou-se como definição de falha de indução, a falta de identificação da fase ativa do trabalho de parto depois de 36 horas, diferente da empregada neste estudo. Talvez por isso a falha de indução tenha sido a indicação mais frequente de cesárea observada por esses autores ${ }^{10}$.

Iniciou-se a indução do parto com uma dose de $20 \mu \mathrm{g} / \mathrm{h}$ de misoprostol oral por seis horas, diferentemente da literatura que utilizou a mesma dose por um período de quatro horas ${ }^{10}$. Neste estudo, na maioria das pacientes $(60 \%)$, o trabalho de parto foi induzido satisfatoriamente com a dose inicial de $20 \mu \mathrm{g} / \mathrm{h}$, não havendo necessidade de se aumentar a dose para $40 \mu \mathrm{g} / \mathrm{h}$, ou seja, a maioria das pacientes iniciou o trabalho de parto nas primeiras seis horas de indução. No estudo de Cheng et al. ${ }^{10}$, esse resultado não foi descrito ${ }^{10}$. Descreve-se que $75 \%$ da pacientes tiveram o parto vaginal em até 12 horas, que a mediana da dose da solução oral foi significativamente maior que o grupo vaginal (180 $\mu \mathrm{g}$ versus $50 \mu \mathrm{g} ; \mathrm{p}<0,01)$ e que a dosagem total da solução oral variou de $40 \mu \mathrm{g}$ a $1.120 \mu \mathrm{g}^{10}$.

Quanto aos efeitos adversos, no único estudo semelhante encontrado, observou uma frequência menor de taquissistolia, apenas $6,9 \%$, com o uso da solução oral de misoprostol ${ }^{10}$. Enquanto neste estudo a taquissistolia ocorreu em 13,3\%. Em ambos os estudos não foram encontrados hipertonia e nem síndrome da hiperestimulação uterina. No entanto, no presente estudo, houve necessidade de droga tocolítica nas pacientes que desenvolveram taquissistolia, à diferença da literatura, em que apenas medidas de ressuscitação foram suficientes para relaxamento uterino ${ }^{10}$. De qualquer forma, como no estudo de Cheng et al. ${ }^{10}$ descreve-se o aumento da dose em menor tempo que o 
nosso (quatro versus seis horas) e uma elevada frequência de parto vaginal em 12 horas (75\%), não foi possível explicar a diferença encontrada na taxa de taquissistolia deste estudo, salvo pelo pequeno tamanho da amostra. Cumpre destacar que essa taxa está bem próxima à descrita pela revisão sistemática da biblioteca Cochrane ${ }^{4} \mathrm{e}$ a outros estudos que utilizaram a via sublingual do misoprostol, em torno de $12 \%{ }^{8,11}$.

Quanto aos desfechos perinatais, Cheng et al. ${ }^{10}$ não avaliaram a presença de mecônio no líquido amniótico ${ }^{10}$, enquanto neste estudo o mecônio foi encontrado em seis pacientes $(20 \%)$. Outros estudos encontraram uma frequência de mecônio de $16 \%$ na via sublingual, $17 \%$ na vaginal ${ }^{13}$ e $14 \%$ na oral ${ }^{14}$. Escores de Apgar $<7$ no primeiro minuto foram observados em dois recém-nascidos do presente estudo $(6,7 \%)$, enquanto Cheng et al. ${ }^{10}$ não encontraram nenhum caso de baixos escores de Apgar em seu estudo. Outros estudos observaram diferentes frequências de escore de Apgar < 7, variando de 13 a 15\%, com a via sublingual e $14 \%$ com a via vaginal ${ }^{8,13}$.

Em suma, comparando o presente estudo ao único encontrado na literatura sobre a solução oral escalonada de misoprostol, conclui-se que estes resultados não foram tão animadores quanto ao estudo de Cheng et al. ${ }^{10}$, porém essa nova forma de administração parece efetiva e segura para a indução do trabalho de parto em gestações com fetos vivos e a termo. Ensaios clínicos randomizados posteriores comparando essa formulação com o uso do comprimido oral em dose fixa, bem como com o uso de misoprostol vaginal, são ainda necessários antes de se estabelecer o seu uso definido na prática clínica diária. Ressalta-se que outros ensaios clínicos estão sendo realizados para comparar essa forma escalonada oral de administração do misoprostol a via vaginal.

\section{Referências}

1. Tang OS, Gemzell-Danielsson K, Ho PC. Misoprostol: pharmacokinetic profiles, effects on the uterus and side-effects. Int J Gynaecol Obstet. 2007;99 Suppl 2:S160-7.

2. Margulies M, Voto LS, Catuzzi P, Imaz FU. Inducción del trabajo de parto con un análogo de la $\mathrm{PgE}_{1}$. Prensa Med Arg. 1991;78(1):9-13.

3. Hofmeyr GJ, Gülmezoglu AM. Vaginal misoprostol for cervical ripening and induction of labour. Cochrane Database Syst Rev. 2010;(1):CD000941.

4. Alfirevic Z, Weeks A. Oral misoprostol for induction of labour. Cochrane Database Syst Rev. 2010;(3):CD001338.

5. Muzonzini G, Hofmeyr GJ. Buccal or sublingual misoprostol for cervical ripening and induction of labour. Cochrane Database Syst Rev. 2010;(1):CD004221.

6. American College of Obstetricians and Gynecologists. ACOG committee opinion Number 228. Induction of labor with misoprostol. Washington, DC: ACOG; Nov. 1999.

7. Royal College of Obstetricians and Gynaecologists. Induction of labour [Evidence-based clinical guideline; no. 9]. London: RCOG Press; 2001

8. Souza AS, Amorim MM, Feitosa FE. Comparison of sublingual versus vaginal misoprostol for the induction of labour: a systematic review. BJOG. 2008;115(11):1340-9.
9. Tang OS, Schweer H, Seyberth HW, Lee SW, Ho PC. Pharmacokinetics of different routes of administration of misoprostol. Hum Reprod. $2002 ; 17(2): 332-6$.

10. Cheng SY, Ming $H$, Lee JC. Titrated oral compared with vaginal misoprostol for labor induction: a randomized controlled trial. Obstet Gynecol. 2008; 111 (1):119-25.

11. Feitosa FEL, Amorim MMR, Alencar Júnior CA, Coutinho IC, Sampaio ZS. Nova formulação de misoprostol sublingual na indução do trabalho de parto. Rev Assoc Med Bras. 2006;52(4):251-5.

12. Range-Filho FA, Alencar Júnior CA, Feitosa FEL, Carvalho FHC, Arcanjo FCN. Baixas doses de misoprostol vaginal $(12,5$ versus $25 \mathrm{mcg}$ ) para indução do parto a termo. Rev Bras Ginecol Obstet. 2007;29(12):639-46.

13. Feitosa FE, Sampaio ZS, Alencar CA Jr, Amorim MM, Passini R Jr. Sublingual versus vaginal misoprostol for induction of labor. Int J Gynaecol Obstet. 2006;94(2):91-5.

14. Kundodyiwa TW, Alfirevic Z, Weeks AD. Low-dose oral misoprostol for induction of labor: a systematic review. Obstet Gynecol. 2009; $113(2 \mathrm{Pt} 1): 374-83$. 